\title{
Clinical Experience of Aortic Valve Surgery in Patients With Aortitis Disease
}

\author{
Yunxing Xue, MD, Jun Pan, $\mathrm{PhD}$, MD, Qing Zhou, MD, PhD, Qiang Wang, MD, PhD, \\ Hailong Cao, MD, PhD, Fudong Fan, MD, Dongjin Wang, MD, PhD \\ Department of Thoracic and Cardiovascular Surgery, The Affiliated Drum Tower Hospital of Nanjing University Medical School, \\ Nanjing, China; Department of Thoracic and Cardiovascular Surgery, Drum Tower Clinical Medical College of Nanjing Medical \\ University; Institute of Thoracic and Cardiovascular Diseases of Nanjing University
}

\section{ABSTRCT}

Objective: To investigate single center's clinical experiences of aortic valve surgery for aortitis patients.

Methods: From January 2010 to December 2018, 15 patients with aortitis disease were treated in our center. Among them, there were 7 males and 8 females with an average age of $48.5 \pm 13.9$ (29-76) years. Six patients were diagnosed as giant cell arteritis, 2 as Takayasu arteritis (Arteritis Group, $\mathrm{N}=8$ ) and 7 as Behcet's disease (BD Group, $N=7$ ). Aortic valve surgery includes valvuloplasty, valve replacement, and root replacement (Bentall procedure).

Results: There were 15 cases with 19 operations, 8 cases in the Arteritis Group received 8 operations while 7 cases in the BD Group received 11 operations, including 4 redo operations. Preoperative patients' aortic valve regurgitation degree, diameter of ascending aorta and left ventricular ejection fraction were similar between the two groups. The type of aortic valve surgery also was no different. Cardiopulmonary bypass time of the BD Group seemed longer than the Arteritis Group but no different, the same as clamp time. Mechanical ventilation time is longer in the BD Group. The morbidity and mortality were similar, but the BD Group had significantly higher incidence of redo operations because of postoperative paravalvular leak or valve insufficiency (Arteritis Group versus BD Group, 0\% versus $57.1 \%, P=.026$ ).

Conclusions: Clinical diagnosis and management of aortic valve patients with arteritis require comprehensive considerations. For aortitis patients with aortic valve surgery, special surgical techniques can be used to reduce the risk of prosthetic valve detachment.

\section{INTRODUCTION}

Aortitis is a common disease concomitant with aortic valve lesion, while the auto-immune process has determined late dilation of the aorta and complications such as valve detachment and heart failure. Surgeons focus on perioperative management of aortitis patients with aortic valve disease,

Received fune 9, 2019; accepted August 5, 2019.

Correspondence: Dongjin Wang, MD, PbD, The Affiliated Drum Tower Hospital of Nanjing University Medical School, 321 Zhongshan Road, Nanjing 210000, Fiangsu, China; +86-13915980346 (e-mail: dongjinwang_gl@163.com). including surgical strategy, special operative methods and immune therapy, but the risks of re-operating procedures due to valve detachment and heart failure-related mortality still are major limits for most cases. There are no clear guidelines for aortitis patients. Our cases can inform and provide different management strategies. Here, we introduce our center's experiences with therapeutic process, results, and special operative methods of aortic valve surgery for aortitis patients.

\section{METHODS}

Patients: From January 2010 to December 2017, 15 patients with aortitis disease underwent aortic valve surgery in our center. Among these patients, 6 patients were diagnosed with giant cell arteritis, and 2 patients were diagnosed with Takayasu arteritis. We divided these 8 patients into the Arteritis Group. The other -- BD Group -- included 7 patients with Behcet's disease. There were 19 aortic valve operations for these 15 patients with 4 redo operations. Figure 1 illustrates the treatment process for these 15 patients.

Intra-operative pathological findings of lymphocyte infiltration and myxoid degenerations are typical characteristics of aortitis disease. But clinical diagnosis of aortitis disease was confirmed through clinical manifestations, symptoms and signs, imaging findings, serum inflammatory indicators (CRP and ESR), and pathological appearances. GCA and Takayasu arteritis are diagnosed according to American College of Rheumatology diagnostic criteria [Hunder 1990; Arend 1990]. BD is characterized by the clinical triad of oral ulcers, genital ulcers, and uveitis.

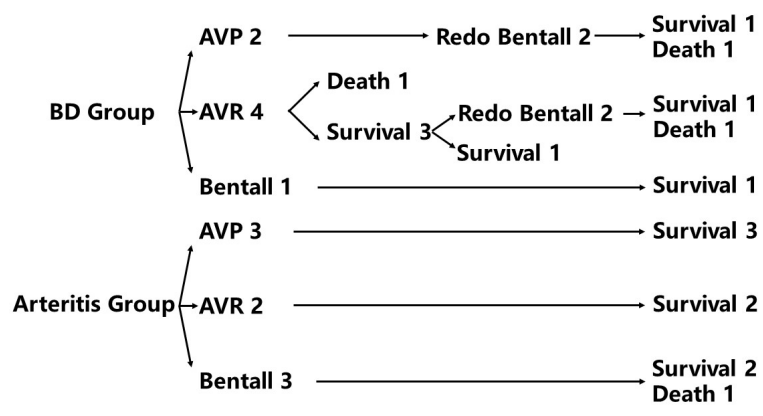

Figure 1. All patients' treatment process. 


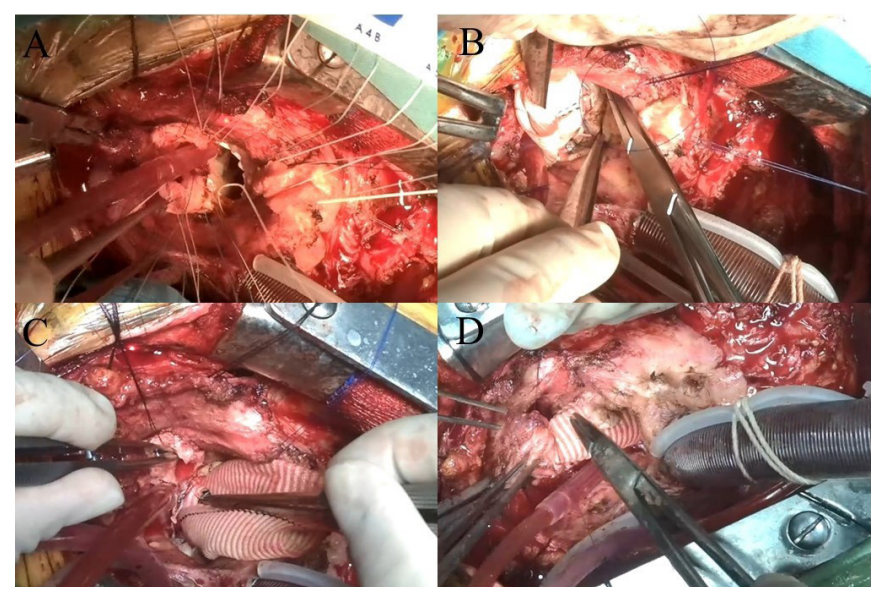

Figure 2. Intraoperative appearances. A. Needle is placed from the outside of the aortic annulus and the pledget is placed outside the aortic wall. B. For Bentall procedure, one more layer of continuous suture connecting the prosthetic valve and pericardial tissue is added. C. Reimplantation anastomosis of coronary artery ostium. D. Distal anastomosis of prosthetic vessel and native aorta.

Surgical approach: Three types of aortic valve surgery were applied to these patients. Aortic valve valvuloplasty was used for patients with regurgitation from valve prolapse; also, the annulus and sinus were normal. Valve replacement with a prosthetic valve was used for damaged leaflets that could not achieve good motion through valvuloplasty. For patients with an insufficient aortic valve and dilated aortic root, Bentall procedure was preferred. In four cases, Bentall procedure had to be reperformed.

During the process of aortic valve replacement and Bentall procedure, the needle is placed from the outside of the aortic annulus, and the pledget placed outside the aortic wall. The native aortic wall is reinforced through a sandwich structure between the suture ring of the prosthetic valve and pledgets outside the aortic wall. Furthermore, one more suture layer connecting the prosthetic suture ring and native residual tissue is applied for Bentall procedure (Figure 2).

We followed up with all patients. Echocardiography and serum inflammation indicators are the main indicators of observation. For patients with a para-valvular leak or prosthetic valve dehiscence, a redo of the operation is recommended.

Statistical analysis: Continuous variables are stated as mean \pm standard deviation. Categorical variables are stated as absolute numbers and proportions. Data analysis was performed using SPSS 22 Statistics software (IBM, Herrenberg, Germany). Differences in categorical variables were analyzed using the $\chi^{2}$ test. Differences in continuous variables were tested using t-test or Mann-Whitney U-test, respectively. A value of $P<.05$ was considered statistically significant.

\section{RESULTS}

Baseline data: The patient average age was $48.5 \pm 13.9$ years (29-76 years), the male percentage was $46.7 \%(7 / 15)$.

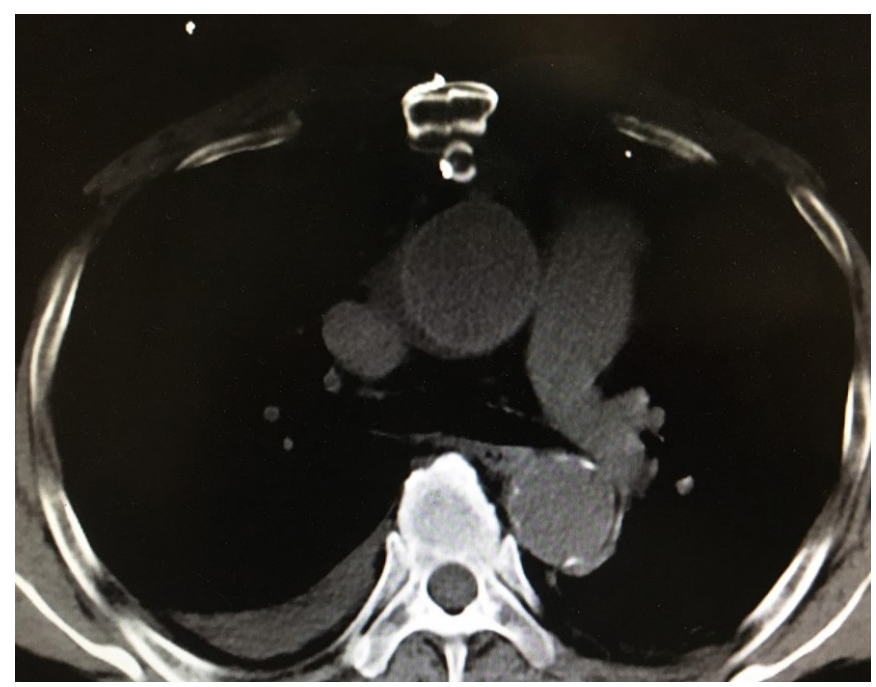

Figure 3. CT showing reveals the aortic wall annular calcification.

No difference existed between the Arteritis Group and BD Group. Aortic regurgitation was the main pathophysiological manifestation. All patients showed aortic dilation and decreased cardiac function (Tables 1 and 4).

Diagnostic confirm was completed by a physician, pre-op clinical or post-op clinical. This means 2 patients in the Arteritis Group were confirmed by a rheumatologist while 5 patients were confirmed in the BD group. Six patients in the Arteritis group were diagnosed before surgery and intra-operative pathological findings confirmed, while only 2 patients in the BD Group were diagnosed before surgery and after admission because of heart dysfunction symptoms. Only 1 patient in the Arteritis Group was confirmed by intraoperative pathological findings without any suspect before surgery (Table 1).

Operative data: There was no significant difference in the surgical methods between the two groups, while Bentall procedure was more common in the Arteritis Group and single AVR was popular with the BD Group. CPB and aortic clamp time was longer in the BD Group, although there was no significant difference.

Postoperative data and complications: Mechanical ventilation time and intensive care time significantly were longer in the BD Group, possibly associated with more redo-surgery patients and more difficult surgical procedures. Re-exploration, stroke, mediastinal infection, and pulmonary infection appeared as major complications post-operation.

Follow up: All patients underwent rigorous follow-up observation. During the follow-up period, the main indicators of inflammation, CT images, and cardiac ultrasound were observed. Four patients in the BD Group received redo Bentall procedures during this period. Two of them had aortic valvuloplasty in the first-stage procedure and 2 patients received mechanical prosthetic valve replacement. The mortality of the redo procedure was 50\% (2/4). Postoperative immunosuppressive therapy is mainly based on the level of inflammation. 
Table 1. Clinical characteristics

\begin{tabular}{|c|c|c|c|c|c|c|}
\hline 1 & 56 & Female & GCA & 1 & Pre-op clinical & No \\
\hline 3 & 52 & Female & GCA & 1 & Pre-op clinical & Yes \\
\hline 4 & 29 & Male & Takayasu & 1 & Pre-op clinical & Yes \\
\hline 6 & 70 & Male & GCA & 1 & Physician & Yes \\
\hline 7 & 59 & Female & GCA & 1 & Pre-op clinical & No \\
\hline 8 & 39 & Male & GCA & 1 & Pre-op clinical & Yes \\
\hline 9 & 46 & Male & $\mathrm{BD}$ & 1 & Physician & No \\
\hline 10 & 31 & Male & $\mathrm{BD}$ & 2 & Pre-op clinical & Yes \\
\hline 14 & 76 & Female & $\mathrm{BD}$ & 1 & Physician & No \\
\hline 15 & 39 & Male & $\mathrm{BD}$ & 1 & Physician & No \\
\hline
\end{tabular}

Table 2. Clinical manifestations and pathologic appearances of the Arteritis Group

\begin{tabular}{cccccc}
\hline Num & Symptoms & $\begin{array}{c}\text { Limb blood } \\
\text { pressure difference }\end{array}$ & $\begin{array}{c}\text { Serum inflammation } \\
\text { biomarkers }\end{array}$ & Imaging performance & Intraoperation pathologic appearances \\
\hline 1 & Heart dysfunction & No & Normal & Yes & Degenerative change Atherosclerotic change \\
2 & Heart dysfunction & No & Normal & No & Inflammatory infiltration Mucoid degeneration \\
Degenerative change & & & Elevation & No & Inflammatory infiltration \\
3 & Heart dysfunction & No & Normal & Yes & Degenerative change Atherosclerotic change \\
4 & Heart dysfunction & Yes & Normal & Yes & Degenerative change Atherosclerotic change \\
5 & Heart dysfunction & Yes & Normal & No & Inflammatory infiltration Mucoid degeneration \\
7 & Heart dysfunction & No & Degenative change Atherosclerotic change \\
8 & Heart dysfunction & Yes & Yes & Degenerative change Atherosclerotic change
\end{tabular}

\section{DISCUSSION}

Diagnosis of aortitis: According to epidemiology study, the incidence of GCA is $1.5-18.8$ per 100,000 per year [Salvarani 2004; Kobayashi 2003], and the incidence of Takayasu is 0.41.0 per 1,000,000 per year [Lane 2005; Reinhold-Keller 2005]. But in a Cleveland Clinic case series, the prevalence of aortitis on clinically diagnosed inflammatory disease was $3.7 \%$ and on surgical pathology specimens was $2.0 \%$, which is much higher than a population study [Svensson 2015]. Based on our center's volume, we treat 2,000 to 3,000 cardiovascular cases every year. There will be 40 to 60 cases of aortitis, but the real case volume is limited. The possible reasons for this include lack of foundation for clinically suspected diagnosis, lack of diagnostic evidence, and non-specific pathological inflammation findings.
Although arteritis (both Takayasu and GCA) has specific diagnostic criteria, diagnosis is more dependent on pathological outcomes. All patients in the Arteritis Group were admitted due to heart dysfunction. Four patients (4/8) were experiencing limb blood pressure difference and two others showed clinical image manifestations. The classical CT manifestation revealed the aortic wall annular calcification (Figure 3). One other had elevated levels of CRP and ESR, while the last one had no significant positive findings before surgery and was diagnosed through intra-operative pathological findings (Figure 4).

The patients in the BD Group underwent a different clinical diagnosis process. Oral ulcer was expressed for every case, and triad symptoms were common, which induced confirmed diagnosis by the physicians or surgeons prior to surgery (Table 3 ). 
Table 3. Clinical manifestations and pathologic appearances of the BD Group

\begin{tabular}{lccccccc}
\hline Num & $\begin{array}{c}\text { Appearance of } \\
\text { BD }\end{array}$ & $\begin{array}{c}\text { Serum inflammation } \\
\text { biomarkers }\end{array}$ & $\begin{array}{c}\text { Imaging } \\
\text { performance }\end{array}$ & Oral ulcer & $\begin{array}{c}\text { Genital } \\
\text { ulcer }\end{array}$ & Eye & Skin \\
\hline 9 & Yes & No & No & Yes & Elevation & No & Inflammatory infiltration Mucoid degeneration \\
10 & Yes & No & No & Yes & Elevation & Yes & Medical rupture Inflammatory infiltration \\
11 & Yes & Yes & No & Yes & Elevation & Yes & Medical rupture \\
12 & Yes & Yes & No & No & No & No & Mucoid degeneration \\
13 & Yes & No & No & Yes & No & Yes & Inflammatory infiltration \\
14 & Yes & No & No & Yes & No & Yes & Inflammatory infiltration \\
15 & Yes & Yes & No & Yes & No & No & Medical rupture Inflammatory infiltration
\end{tabular}

Table 4. Comparison data between Arteritis Group and BD group

\begin{tabular}{|c|c|c|c|}
\hline Gender (Male, \%) & $3,37.5 \%$ & $4,57.1 \%$ & .619 \\
\hline AR $(0-4)$ & $3.1 \pm 0.99$ & $3.6 \pm 0.79$ & .200 \\
\hline Aorta size $(\mathrm{cm})$ & $43.9 \pm 7.0$ & $47.7 \pm 9.3$ & .393 \\
\hline Surgical approach & - & - & .549 \\
\hline $\operatorname{AVP}(N, \%)$ & $3,37.5 \%$ & $2,28.6 \%$ & \\
\hline $\operatorname{AVR}(N, \%)$ & $2,25.0 \%$ & $4,57.1 \%$ & \\
\hline Bentall (N, \%) & $3,37.5 \%$ & $1,14.3 \%$ & \\
\hline Ascending aorta replacement (N, \%) & $4,50 \%$ & $4,57.1 \%$ & 1.0 \\
\hline Mechanical ventilation $(h)$ & $12.8 \pm 3.4$ & $28.4 \pm 33.2$ & .017 \\
\hline $\mathrm{ICU}(\mathrm{d})$ & $3.8 \pm 2.2$ & $6.4 \pm 4.2$ & .257 \\
\hline \multicolumn{4}{|l|}{ Complications } \\
\hline Re-exploration (N, \%) & 0 & $1,14.3 \%$ & .467 \\
\hline Stroke (N, \%) & $1,12.5 \%$ & $1,14.3 \%$ & 1.0 \\
\hline Mediastinal infection (N, \%) & $1,12.5 \%$ & $1,14.3 \%$ & 1.0 \\
\hline Pulmonary infection (N, \%) & $1,12.5 \%$ & $2,28.6 \%$ & .569 \\
\hline Mortality (N, \%) & $1,12.5 \%$ & $1,14.3 \%$ & 1.0 \\
\hline Paravalvular leak induced redo aortic valve surgery & 0 & $4,57.1 \%$ & .026 \\
\hline
\end{tabular}

But even if we maintained sensitivity to the clinical diagnosis of aortitis, we still may have lost many patients who may be diagnosed. This is also a possible cause of early paravalular leakage and redo surgery in some patients with aortic valve surgery.

Surgical method: Surgical strategy and methods are a major influencing factor for aortitis patients with aortic valve surgery by the clinical studies. In the early stages, we choose valvuloplasty for aortic regurgitation, but 2 patients in the BD Group received a reperformance of Bentall procedures. The mechanism of aortic regurgitation mainly is due to annulus dilation because of inflammation of the aortic wall. Subcommissure valvuloplasty will correct regurgitation, but the lateterm dilation of aortic annulus will induce relapse. From this perspective, valve-sparing root replacement may be effective. 
Aortic valve replacement with prosthetic valve seems to be the first choice for most cases. But prosthetic valve detachment may occur and require a redo operation. Ando et al reported different results in patients with Behçet's disease and Takayasu's arteritis. In their experiences, the rate of redo procedures was $40 \%$ in Behçet's disease ( 4 of $10 ; 40 \%$ ), while the rate was $4.6 \%$ with Takayasu arteritis (3 of 65; 4.6\%) [Ando 1998; Ando 1999]. And the 5 years' and 10 years' rate of free of valve detachment or false aneurysm formation in the suture line Takayasu patients was $96 \%$ and $94 \%$, respectively, but the rate in Behçet's patients was only $64 \%$ and $43 \%$, respectively. In Adachi's study, 2 patients with Takayasu disease needed a redo root replacement with the rate of 50\% [Adachi 2007]. Thus Adachi suggested that lowering the threshold of root replacement may be reasonable in patients with aortitis. The same results were reported by Jeong, which showed event-free survival at 13 years was $39.2 \%$ in an aortic root replacement group, but this was only $4 \%$ in patients who underwent valve replacement $(P=.001)$ [Jeong 2009]. In our cases, we chose aortic valve replacement with a non-dilation root in the early stages, but the rate of redo procedures due to valve detachment was very high. We now prefer root replacement even with a normal root size.

To fix the prosthetic valve at the aortic annulus and prevent valve detachment, we put buttress sutures from the outside of the aortic wall with pledgets outside. And in the cases of root replacement, we also put one more layer of continuous suture between the sutured ring of the prosthetic valve and pledgets. This special method was introduced by several articles. Tang reported a similar method, but the suture line was above the annulus [Tang 2012]. We were concerned with the risk of coronary artery injury and the following dilation of remaining aortic wall, but no follow-up results were reported in Tang's paper. Another kind of surgical method is subannular sutures [Azuma 2009; Chen 2017]. Chen checked the pathological manifestations of the aortic wall and myocardium of the left ventricle outflow tract, and the results revealed no inflammation involving the myocardium, which lowered risk of secondary dilation after a first-stage operation. But in our opinions, myocardium is more fragile than the annulus, and we still consider whether myocardium is strong enough. The second risk is the postoperative heart block, which showed the rate of $42.9 \%$ in Jung's study [Jung 2017]. No matter the method selected, the perioperative results show no significant difference. Otherwise, we all know regular aortic valve replacement method is not suitable for patients with aortitis.

Immunotherapy: Jeong reported event-free survival at 13 years in patients who were administered immunosuppressive therapies was $33.7 \%$; the rate was $0 \%$ in patients not administered immunosuppressive therapy. The elevated level of inflammatory biomarkers is the indicator of immunosuppressive therapy after surgery, but no evidence can prove immunotherapy can reduce the rate of valve detachment. Furthermore, the specific immunosuppressive treatment programs do not have a clear unified plan [Saleh 2014].

Limitations: This is our center's experience with a low volume of cases. We believe these cases with aortitis patients with aortic valve surgery are important and worthy for others to compare results.

\section{CONCLUSION}

Aortic valve surgery for aortitis patients is still a big challenge for cardiovascular surgeons because it comes with a high risk of valve detachment and heart failure, despite progression of immune therapy and operative methods. Aortic valvuloplasty is not effective for most cases, and single aortic valve replacement can achieve similar results in selected cases. But more invasive surgery, such as root replacement in low threshold patients and special methods like a non-usual level for the aortic prosthetic valve ring or reinforcement using plegets outside the aortic wall are reasonable and effective. No clear recommendations for immune therapy have been stated, but elevated levels of biomarkers can be an indictor for aggressive immune therapy.

\section{REFERENCES}

Adachi O, Saiki Y, Akasakaa J, et al. 2007. Surgical management of aortic regurgitation associated with Takayasu arteritis and other forms of aortitis. Ann Thorac Surg 84:1950-4.

Ando M, Kosakai Y, Okita Y, et al. 1998. Surgical treatment for aortic regurgitation caused by Takayasu's arteritis. J Card Surg 13:202-7.

Ando M, Kosakai Y, Okita Y, et al. 1999. Surgical treatment of Behçet's disease involving aortic regurgitation. Ann Thorac Surg 68:2136-40.

Arend WP, Michel BA, Bloch DA, et al. 1990. The American College of Rheumatology 1990 criteria for the classification of Takayasu arteritis. Arthritis Rheum. 33(8):1129-34.

Azuma T, Yamazaki K, Saito S, Kurosawa H. 2009. Aortic valve replacement in Behcet's disease: surgical modification to prevent valve detachment. Eur J Cardiothorac Surg. 2009 Oct;36(4):771-2.

Chen LW, Wu XJ, Cao H, Dai XF. 2017. Valved Conduit Attached to Left Ventricular Outflow Tract for Valve Detachment in Behçet's Disease. Ann Thorac Surg. Mar;103(3):e301-e303.

Hunder GG, Bloch DA, Michel BA, et al. 1990. The American College of Rheumatology 1990 criteria for the classification of giant cell arteritis. Arthritis Rheum. 33(8):1122-8.

Jeong DS, Kim KH, Kim JS, Ahn H. 2009. Long-term experience of surgical treatment for aortic regurgitation attributable to Behçet's disease. Ann Thorac Surg. Jun;87(6):1775-82.

Jung Y, Ahn BH, Lee KS, et al. 2017. A novel solution to prosthetic valve dehiscence after aortic valve surgery in Behçet's disease. Interact Cardiovasc Thorac Surg. Mar 1;24(3):342-347.

Kobayashi S, Yano T, Matsumoto Y, et al. 2003. Clinical and epidemiologic analysis of giant cell (temporal) arteritis from a nationwide survey in 1998 in Japan: the first government-supported nationwide survey. Arthritis Rheum. 49:594-598.

Lane SE, Watts R, Scott DG. 2005. Epidemiology of systemic vasculitis. Curr Rheumatol Rep. 7:270 -275.

Reinhold-Keller E, Herlyn K, Wagner-Bastmeyer R, Gross WL. 2005. Stable incidence of primary systemic vasculitides over five years: results from the German vasculitis register. Arthritis Rheum. 53:93-99.

Salvarani C, Crowson CS, O'Fallon WM, et al. 2004. Reappraisal of the epidemiology of giant cell arteritis in Olmsted County, Minnesota, over a fifty-year period. Arthritis Rheum. 51: 264-268. 
Svensson LG, Arafat A, Roselli EE, et al. 2015. Inflammatory disease of the aorta: patterns and classification of giant cell aortitis, Takayasu arteritis, and nonsyndromic aortitis. J Thorac Cardiovasc Surg. Feb;149(2 Suppl):S170-5.

Tang Y, Xu Z, Liao Z, Xu J. 2012. Supraannular aortic replacement for severe valve detachment attributable to Behçet's disease. Ann Thorac Surg. Aug;94(2):e55-7.

Saleh Z, Arayssi T. 2014. Update on the therapy of Behçet disease. Ther Adv Chronic Dis. May;5(3):112-34. 